\title{
Triplet Probe Study of Collision Rates of Small Molecules with Chain Terminal and Central Groups of Polystyrene
}

\author{
Kazuyuki Horie, Keiko TOMOMUne, and Itaru MitA \\ Institute of Space and Aeronautical Sciences, University of Tokyo, \\ Komaba, Meguro-ku, Tokyo 153, Japan.
}

(Received December 4, 1978)

\begin{abstract}
To study the mechanism of collision rates between small molecules and polymers, the rate constants $k_{\mathrm{q}}$ for quenching of phosphorescence of benzil by a terminal or central anthryl group attached to polystyrene (degree of polymerization, $P=25-10,000$ ) have been measured in benzene, butanone, and cyclohexane at $20-40^{\circ} \mathrm{C}$ by using a $10 \mathrm{~ns}$ dye laser pulse. The reduced rate constant $k_{\mathrm{q}} \eta_{0} / T$ for quenching of benzil by a terminal anthryl group of polystyrylanthracene decreases to $1 / 2-1 / 3$ of that for quenching of benzil by 9 -methylanthracene. No difference in $k_{\mathrm{q}} \eta_{0} / T$ due to the difference in solvent was observed for this case. The curve of $k_{\mathrm{q}} \eta_{0} / T$ for quenching of benzil by the central anthryl group of PS-A-PS against $P$ shows an additional decrease in $k_{\mathrm{q}} \eta_{0} / T$ for $P>10^{3}$. This decrease is more marked in poor solvents than in a good solvent. These results are explained consistently by considering the obstructive effect of polymer segments on the diffusion of the reacting benzil molecule through the polymer coil.
\end{abstract}

KEY WORDS Rate Constant / Collision / Diffusion Control / Polymer Reaction / Phosphorescence / Quenching / Triplet Probe / Laser Pulse / Benzil / Polystyrylanthracene /

The collision of a small molecule with a functional group attached to the polymer chain is the first elementary step of enzymatic and other polymeric reactions. Measurements of the collision rates for flexible polymers have been carried out by fluorescence quenching, ${ }^{1,2}$ triplet quenching, ${ }^{3,4}$ and pulse radiolysis technique. ${ }^{5,6}$ Fluorescence quenching reflects the collision of the functional group on the polymer chain with small molecules only in its immediate vicinity due to the extremely short lifetime of the excited singlet state. The pulse radiolysis technique measures the collision rate of the spherical surface of a polymer coil with a small molecule from outside the polymer coil, but the reactive site along the polymer chain is difficult to specify. In contrast to these techniques, the quenching of a triplet probe with long lifetime provides the measurements of collision rates of a definite active site of a polymer chain with small molecules in very low concentration, as has been shown in our previous paper. ${ }^{4}$ The triplet probe is also effective for the measurements of diffusion-controlled rates of intermacromolecular reactions ${ }^{7}$ and intramolecular collision between a pair of terminal groups of a polymer chain. ${ }^{8}$

In the previous work, ${ }^{4}$ quenching rates of phosphorescence of benzil by the anthryl group attached to a chain end of polystyrene (PS-A) were measured in benzene for the range of degree of polymerization, $P=25-740$. In the present work, polystyrene having an anthryl group at the center of its chain (PS-A-PS) was prepared and quenching rates of benzil phosphorescence by PS-A, A-PS-A, and PS-A-PS were measured in various solvents at 20 $40^{\circ} \mathrm{C}$ for $P=25-10,000$. Some discussion will be given about the difference in collision rates caused by the location of the anthryl group in the polymer chain.

\section{EXPERIMENTAL}

Polystyrenes with anthryl groups at one chain end (PS-A) and at both chain ends (A-PS-A) were prepared by the reactions of 9-chloromethylanthracene with living polystyryl monoanion ${ }^{4}$ and dianion, ${ }^{8}$ respectively. The direct attack of living polystyryl monoanion ( $\mathrm{PS}^{-}$) prepared with cumyl potassium in tetrahydrofuran ${ }^{9}$ with a stoichiometric 
amount of 9,10-bis(chloromethyl)anthracene ${ }^{10}$ in a high vacuum system gave polystyrene with an anthryl group in the center of its chain (PS-A-PS). The number-average molecular weights $\bar{M}_{n}$ for PS-A-PS were proved to be about twice as high as $\bar{M}_{n}$ for the corresponding PS ${ }^{-}$by GPC measurements, while molecular weight distributions $\bar{M}_{w} / \bar{M}_{n}$ for both PS-A-PS and the original PS ${ }^{-}$were rather broad, probably due to the incomplete mixing of initiator solution with monomer solution within the time scale of the initiation reaction at the time of the preparation of $\mathrm{PS}^{-}$. So, the PS-A-PS used for phosphorescence measurements was fractionally precipitated from a benzene-methanol system.

Table I shows the values of $\bar{M}_{n}$ and $\bar{M}_{w} / \bar{M}_{n}$ measured with a GPC (Toyo Soda HLC-802UR), as well as the percentages of anthryl group in the polymers relative to its theoretical content which was measured with an ultraviolet spectrophotometer (Shimadzu MPS-5000).

Table I. Characterization of polystyrylanthracene

\begin{tabular}{lrccc}
\hline \multicolumn{1}{c}{ Polymer } & $P$ & $\bar{M}_{n}$ & $\bar{M}_{w} / \bar{M}_{n}$ & $\begin{array}{c}\% \\
\% \\
\text { group }\end{array}$ \\
\hline PS-A & 25 & $2.8 \times 10^{3}$ & 1.03 & 100 \\
& 71 & $7.5 \times 10^{3}$ & 1.13 & 96 \\
& 270 & $2.9 \times 10^{4}$ & 1.17 & 90 \\
A-PS-A & 740 & $7.7 \times 10^{4}$ & 1.14 & 61 \\
& 1300 & $1.4 \times 10^{5}$ & 1.42 & 100 \\
& 3000 & $3.1 \times 10^{5}$ & 1.24 & 91 \\
PS-A-PS & 10000 & $1.0 \times 10^{6}$ & 1.09 & 15 \\
& 60 & $7.0 \times 10^{3}$ & 2.41 & 63 \\
& 210 & $2.3 \times 10^{4}$ & 1.60 & 24 \\
& 730 & $7.6 \times 10^{4}$ & 2.40 & 86 \\
& 2500 & $2.8 \times 10^{5}$ & 1.82 & 100 \\
& 3200 & $3.3 \times 10^{5}$ & 1.39 & 95 \\
\hline
\end{tabular}

Dotite-Luminasol grade benzene and cyclohexane and Dotite-Spectrosol grade butanone were used as solvents for phosphorescence measurements.

The bimolecular rate constant of phosphorescence quenching $k_{\mathrm{q}}$ was calculated by using

$$
1 / \tau=1 / \tau_{0}+k_{\mathrm{q}}[\mathrm{A}]
$$

where $\tau$ and $\tau_{0}$ are triplet lifetimes of phosphorophore in the presence and absence of quencher, respectively, and $[\mathrm{A}]$ is the concentration of anthryl groups. The lifetimes $\tau$ and $\tau_{0}$ were obtained from decay curves of phosphorescence intensity $(560 \mathrm{~nm})$ induced by a dye laser pulse $(406 \mathrm{~nm})$ with a half- width of $10 \mathrm{~ns}$. The apparatus and procedure for measurements were the same as previously reported. ${ }^{4}$ The percentages of error for $k_{\mathrm{q}}$ were within $10 \%$ in most cases, but happened to reach about $20 \%$ in a few cases.

\section{RATE CONSTANTS BETWEEN BENZIL AND PS-A, A-PS-A, OR PS-A-PS}

The rate constants $k_{\mathrm{q}}$ for quenching of benzil by the terminal anthryl group of PS-A or A-PS-A are shown in Figure 1 against the degree of polymerization $P$ of quenchers. The values $k_{\mathrm{q} 0}$ for $P=1$ are the results for benzil and 9-methylanthracene, which is a low-molecular-weight analog for PS-A.

The value of $k_{\mathrm{q}}$ in each solvent decreases with increasing $P$ of polystyrene moiety up to $P \simeq 10^{2}$, then it approaches a constant value which is about $1 / 2-1 / 3$ of the corresponding $k_{\mathrm{q} 0}$. The rate constant in cyclohexane at $20^{\circ} \mathrm{C}$ for $P>10^{3}$ could not be measured since the solution became turbid at that temperature. The rate constant $k_{\mathrm{q}}$ in butanone is larger and that in cyclohexane is smaller than the corresponding $k_{\mathrm{q}}$ in benzene, corresponding to the difference in viscosities of these solvents. These results for the diffusion-controlled reaction between benzil and the chain terminal anthryl group can be well explained by the model described previously, ${ }^{4}$ where the diffusion of a small molecule is supposed to be the determining factor for the reaction. The difference between PS-A and A-PS-A has no influence on the rate constants when they are calcu-

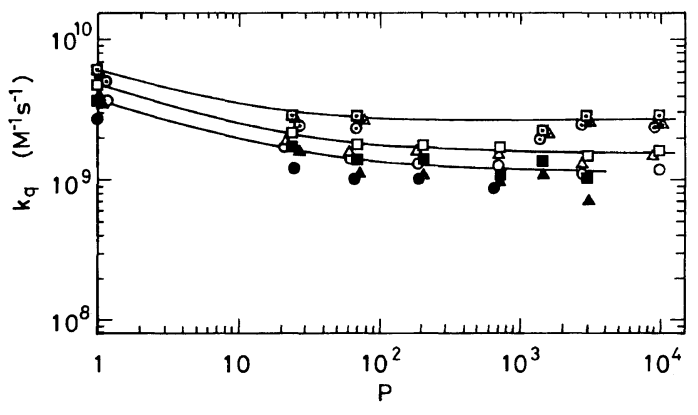

Figure 1. Dependence of $k_{\mathrm{q}}$ for reactions of benzil with PS-A $\left(P<10^{3}\right)$ and A-PS-A $\left(P>10^{3}\right)$ on the degree of polymerization $P$ in benzene $(\bigcirc, \triangle, \square)$, butanone $(\odot$, $\triangle, \square)$, and cyclohexane $(\boldsymbol{O}, \boldsymbol{\Lambda}, \boldsymbol{\square})$. Temperatures: $\bigcirc$, $\odot, \odot, 20^{\circ} \mathrm{C} ; \triangle, \triangle, \Delta, 30^{\circ} \mathrm{C} ; \square, \bullet, \mathbf{\square}, 40^{\circ} \mathrm{C}$. Solid lines correspond to results at $40^{\circ} \mathrm{C}$. 
lated on the basis of molar concentration of anthryl groups.

The rate constants $k_{\mathrm{q}}$ for quenching of benzil by the central anthryl group of PS-A-PS are shown in Figure 2 against the degree of polymerization $P$ of PS-A-PS. The $k_{\mathrm{q}}$ in each solvent shows a sigmoidal dependence on $P$, and in the case of $P>2 \times 10^{3}$ it becomes quite a bit smaller than the corresponding $k_{\mathrm{q}}$ for the terminal anthryl group. The values $k_{\mathrm{q} 0}$ for $P=1$ in the present case correspond to the reaction of benzil with 9,10-dimethylanthracene, which would be regarded as identical to the corresponding $k_{\mathrm{q} 0}$ for the reaction of benzil with 9-methylanthracene within the range of error. The appreciable deviation in $k_{\mathrm{q} 0}$ for benzil-9,10-dimethylanthracene in cyclohexane at 20 and $30^{\circ} \mathrm{C}$ may be caused by the poor solubility of 9,10-dimethylanthracene in cyclohexane. The activation energies for $k_{\mathrm{q}}$ for reactions of benzil with both terminal and central anthryl groups in polystyrylanthracenes seem to be independent of molecular weights of polymer quenchers, in spite of some scatter due to the influence of errors in $k_{\mathbf{q}}$, and are compared with the temperature dependences of the solvent viscosities $\left(10.6 \mathrm{~kJ} \mathrm{~mol}^{-1}\right.$ for benzene, $8.4 \mathrm{~kJ} \mathrm{~mol}^{-1}$ for butanone, and 11.9 $\mathrm{kJ} \mathrm{mol}^{-1}$ for cyclohexane). ${ }^{11}$

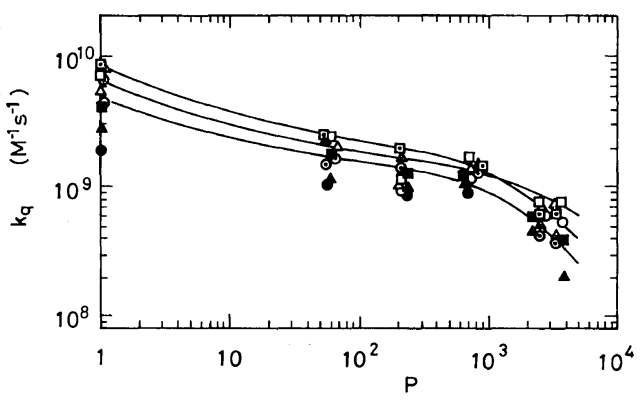

Figure 2. Dependence of $k_{\mathrm{q}}$ for reactions of benzil with PS-A-PS on the degree of polymerization $P$ in various solvents. Symbols are the same as in Figure 1.

In order to remove the influence of solvent viscosity $\eta_{0}$ and reaction temperature $T$ on $k_{\mathrm{q}}, k_{\mathrm{q}}$ is multiplied by $\eta_{0} / T$, since the diffusion-controlled rate constant is expected to be proportional to $T / \eta_{0}{ }^{7}$ The reduced rate constants $k_{\mathrm{q}} \eta_{0} / T$ for reactions of benzil with terminal and central anthryl groups of polystyrylanthracenes are shown in Figure 3 against $P$. As the values of $k_{\mathrm{q}} \eta_{0} / T$ for different $T$ in each solvent agree with each other, they are averaged and expressed as a single point in Figure 3.

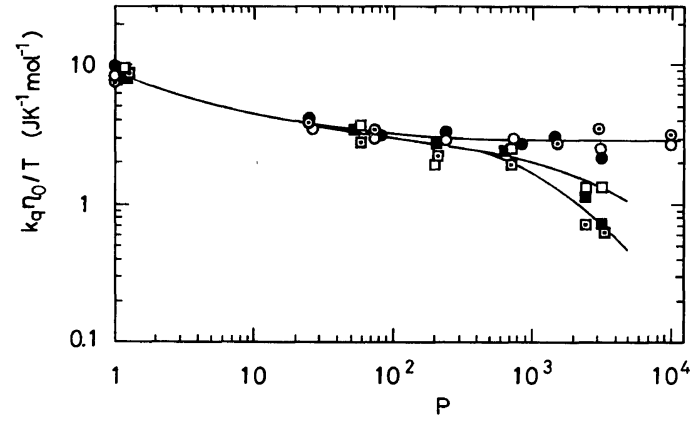

Figure 3. Reduced rate-constants $k_{\mathrm{q}} \eta_{0} / T$ against $P$ for collisions of benzil with a terminal anthryl group $(O, \odot$, -) and the central anthryl group $(\square, \bullet, \boldsymbol{\square})$ of polystyrylanthracenes in benzene $(\odot, \square)$, butanone $(\odot, \odot)$, and cyclohexane

Figure 3 presents the fundamental characteristics of diffusion-controlled reactions of a small molecule with active groups in a polymer chain. When the active group is attached to the end of a polymer chain, the values of $k_{\mathrm{q}} \eta_{0} / T$ in various solvents fit onto a single curve. The difference in coil expansion of PS-A or A-PS-A in various solvents gives little effect on the collision rate of a small molecule with the terminal group of the polymer, even for the case of a very high degree of polymerization $\left(P=10^{4}\right)$. When the active group is attached to the center of the polymer chain, $k_{\mathrm{q}} \eta_{0} / T$ begins to decrease markedly with the increase in $P$ for $P>10^{3}$, and this trend is more pronounced in butanone and cyclohexane (poor solvents) than in benzene (good solvent). A similar solvent effect on rate constants has also been observed for diffusion-controlled polymer-polymer reactions with $P>10^{3} .^{7}$ As the segment density around the anthryl group in a polymer chain is higher in poor solvents than in the good solvent, this decrease in $k_{\mathrm{q}} \eta_{0} / T$ for $P>10^{3}$ is presumably caused by the obstructive effect of polymer segments on the translational diffusion of benzil through the polymer coil.

\section{DISCUSSION}

The comparison of the results for reactions between benzil and PS-A in benzene with a model based on modified Smoluchowski equation 2 has already been given in a previous paper. ${ }^{4}$

$$
k_{\mathrm{q}}=4 \pi \gamma\left(r_{\mathrm{B}}+r_{\mathrm{A}}\right)\left(D_{\mathrm{B}}+D_{\mathrm{A}}\right)
$$


where $r_{\mathrm{B}}$ and $r_{\mathrm{A}}$ are hydrodynamic radii of the benzil and anthryl group, respectively, $D_{\mathrm{B}}$ and $D_{\mathrm{A}}$ are their respective diffusion coefficients, and $\gamma$ is a steric factor. As $D_{\mathrm{A}}$ is comparable to the translational diffusion coefficient for polymer molecule $D_{\mathrm{pt}}$ for the time scale of the present measurements $(10-200 \mu \mathrm{s})$ and $D_{\mathrm{pt}}$ for polystyrene with $P \geqq 25$ is smaller than $D_{\mathrm{B}}$ by more than an order of magnitude, $D_{\mathrm{A}}$ in eq 2 can be neglected in comparison with $D_{\mathrm{B}}$.

The formulation of the diffusion-controlled rate constant including the influence of segment density on the diffusion of benzil molecules is rather difficult, since the microscopic density of polymer segments around a diffusing benzil molecule changes along the path of benzil from outside the polymer coil toward the anthryl group, resulting in the change in $D_{\mathrm{B}}$. Here, instead of setting up and solving a diffusion equation with a reaction term and a potential field caused by segment density, we first evaluate, as a measure of the obstruction against diffusion, the statistical number of monomer units of polystyrylanthracene $v$ existing in the cylindrical path of a benzil molecule from a distant position to the position of the anthryl group, and then examine the dependence of experimental reduced rate constant $k_{\mathrm{q}} \eta_{\mathrm{o}} / T$ on $v$.

The majority of excited benzil molecules B* are supposed to be located outside the polymer coil just after the pulse irradiation due to the low polymer concentration $\left([\mathrm{P}]<4 \mathrm{~g} \mathrm{dm}^{-3}\right)$ and the low $\mathrm{B}^{*}$ concentration $\left(\left[\mathrm{B}^{*}\right]_{0}<10^{-5}<\mathrm{moldm}^{-3}\right)$, and each molecule diffuses toward the anthryl groups at the chain end or the center of polystyrylanthracene. So

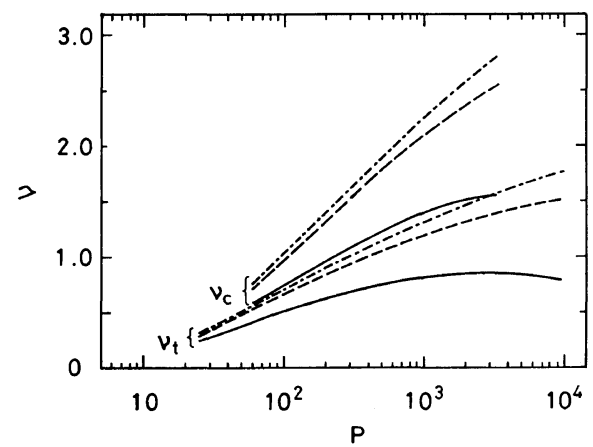

Figure 4. Number of monomer units of polystyrylanthracene $v$ existing in the cylindrical path of a benzil molecule against $P$ in benzene ( - ), butanone ( --$)$, and cyclohexane (-.-). $v_{\mathrm{t}}$, for PS-A and A-PS-A; $v_{\mathrm{c}}$, for PS-A-PS. the values of $v$ for the case of PS-A or A-PS-A $\left(v_{\mathrm{t}}\right)$ and PS-A-PS $\left(v_{\mathrm{c}}\right)$ were calculated by using the following equations, based on a Gaussian distribution for flexible polymer chains, and are shown in Figure 4.

$$
\begin{gathered}
v_{\mathrm{t}}=(1 / 2) \pi r_{\mathrm{B}}^{2} \int_{0}^{\infty}\left\{\rho_{0}+\sum_{k=21}^{2 P} W(k, r)\right\} \mathrm{d} r \\
v_{\mathrm{c}}=\pi r_{\mathrm{B}}^{2} \int_{0}^{\infty}\left\{\rho_{0}+\sum_{k=21}^{P} W(k, r)\right\} \mathrm{d} r \\
W(k, r)=\left(3 / 2 \pi N_{0} k \alpha^{2} b^{2}\right)^{3 / 2} \exp \left(-3 r^{2} / 2 N_{0} k \alpha^{2} b^{2}\right) \\
\rho_{0}=\left\{\begin{array}{cc}
20\left\{(4 / 3) \pi(20 b)^{3}\right\}^{-1} & \text { for } r<3 \mathrm{~nm} \\
0 & \text { for } r>3 \mathrm{~nm}
\end{array}\right.
\end{gathered}
$$

Here $W(k, r)$ is the probability of finding the $k$ th carbon atom in the main chain at the distance $r$ from the anthryl group, and $\rho_{0}$ represents the segment density due to carbon atoms with $k=1-20$, for which the Gaussian distribution does not hold. The critical value $k=20$ was adopted according to the number of monomer units in a segment $N_{0} \cdot{ }^{7}$ The values for $N_{0}$, defined as the mean-square end-to-end distance for an unperturbed chain divided by that for a freely jointed chain $(10.6),{ }^{12}$ for $b$, the carbon-carbon bond length $0.154 \mathrm{~nm}$, and for $r_{\mathrm{B}}$ $0.426 \mathrm{~nm}^{13}$ were used for the calculation together with the values for the coil expansion factor $\alpha$ calculated from data in benzene, ${ }^{14}$ butanone,${ }^{15}$ and cyclohexane. ${ }^{16}$ The slopes of the curves for $v_{c}$ in butanone and cyclohexane in Figure 4 are appreciably larger than those for the other cases.

Semilogarithmic plots for experimental values of $k_{\mathrm{q}} \eta_{0} / T$ against $v$ are shown in Figure 5. The shaded

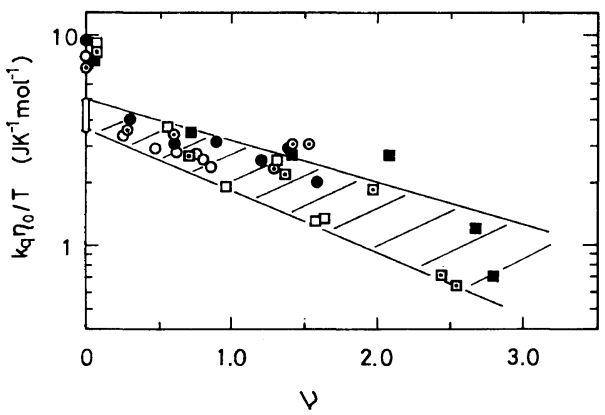

Figure 5. Reduced rate-constants $k_{\mathrm{q}} \eta_{0} / T$ against $v$. Symbols are the same as in Figure 3. 
area, determined so as to include most of the plots, suggests the proportionality of $k_{\mathrm{q}} \eta_{0} / T$ to $\exp (-C v)$ regardless of the position of the anthryl group in PS-A. A-PS-A, or PS-A-PS, where $C$ is a constant. The extrapolation of the shaded area to $v=0$ gives the value of $3.7-5.0$, which agrees with a half $k_{\mathrm{q} 0} \eta_{0} / T$ for $P=1$ where $D_{\mathrm{A}} \simeq D_{\mathrm{B}}$. The above proportionality may be expected if the dependence of the average diffusion coefficient of benzil $\bar{D}_{\mathrm{B}}$ on $v$ is expressed by eq 7 .

$$
\bar{D}_{\mathrm{B}}=D_{\mathrm{B} 0} \exp (-C v)
$$

where $D_{\mathrm{B} 0}$ is diffusion coefficient of benzil in solution without polymer. It should be noted that for $C v \ll 1$, eq 7 is reduced to

$$
\bar{D}_{\mathrm{B}}=D_{\mathrm{B} 0}(1-C v)
$$

which is analogous to expressions ${ }^{17,18}$ for the concentration dependence of the diffusion coefficient of a small molecule in polymer solution. The $D_{\mathbf{B} 0}$ is expressed by

$$
D_{\mathrm{B} 0}=k_{\mathrm{B}} T /\left(6 \pi r_{\mathrm{B}} \eta_{0}\right)
$$

where $k_{\mathrm{B}}$ is Boltzmann's constant. The use of $\bar{D}_{\mathrm{B}}$ in eq 7 in place of $D_{\mathrm{B}}$ in eq 2 , together with $D_{\mathrm{A}} \ll \bar{D}_{\mathrm{B}}$, $r_{\mathrm{A}} \simeq r_{\mathrm{B}}$ and eq 9 , gives

$$
k_{\mathrm{q}}=\left(4 / 3 \eta_{0}\right) \gamma k_{\mathrm{B}} \mathrm{T} \exp (-C v)
$$

Regardless of the position of active group in the polymer chain the reduced rate constant $k_{\mathrm{q}} \eta_{\mathrm{o}} / T$ is, therefore, expressed by

$$
k_{\mathrm{q}} \eta_{\mathrm{o}} / T=(4 / 3) \gamma k_{\mathrm{B}} \exp (-C v)
$$

with $\gamma=0.33-0.45$

Thus, it would be supposed in the first approximation that the difference in $k_{\mathrm{q}} \eta_{0} / T$ for reactions of benzil with anthryl groups at the chain end and at the center of flexible polystyrylanthracene is caused by the difference in segment density of the polystyryl chain, which is considered as an obstacle along the path of benzil toward the reaction with the anthryl group. The magnitudes of $v$ suggest that benzil molecule collides only a few times with the polystyryl chain on its way to the central anthryl group from outside the polymer coil, even for polymers with $P>10^{3}$. The almost free penetration of small molecules inside the polymer coil has also been observed in the case of a diffusion-controlled reaction between small molecules in polymer solution. ${ }^{19}$ However, once the collision occurs, the rate constant is reduced to about one-half for every collision with a polymer chain $(C=0.48-0.67)$, and consequently, $k_{\mathrm{q}}$ for benzil PS-A-PS with $P \simeq 3 \times 10^{3}$ in poor solvents becomes about $4 \times 10^{8}-6 \times 10^{8} \mathrm{~mol}^{-1} \mathrm{dm}^{3} \mathrm{~s}^{-1}$ corresponding to $1 / 17-1 / 10$ of $k_{\mathrm{q} 0}$.

In conclusion, the measurements of collision rates between a small molecule and the terminal or central group of a flexible polymer by the triplet probe method have shown that the reduced rate constants $k_{\mathrm{q}} \eta_{\mathrm{o}} / T$ are affected greatly by the position of the active group in the polymer chain and decrease remarkably in the cases of benzil and PS-A-PS with $P>10^{3}$. This decrease is more marked in poor solvents than in a good solvent. The change in $k_{\mathrm{q}} \eta_{0} / T$ would be caused by the obstructive effect of the polymer chain on the diffusion of the reacting benzil molecule.

\section{REFERENCES}

1. J. P. Bentz, J. P. Beyl, G. Beinert, and G. Weill, Eur. Polym. J., 11, 711 (1975).

2. B. Valeur and L. Monnerie, J. Polym. Sci., Polym. Phys. Ed., 14, 29 (1976).

3. G. Beck, G. Dobrowolski, J. Kiwi, and W. Schnabel, Macromolecules, 8, 9 (1975).

4. K. Horie and I. Mita, Polym. J., 9, 201 (1977).

5. A. Behzadi, U. Borgwardt, A. Henglein, E. Schamberg, and W. Schnabel, Ber. Bunsenges. Phys. Chem., 74, 649 (1970).

6. A. Behzadi and W. Schnabel, Macromolecules, 6, 824 (1973):

7. K. Horie and I. Mita, Macromolecules, 11, 1175 (1978).

8. H. Ushiki, K. Horie, A. Okamoto, and I. Mita, Macromolecules, to be published.

9. K. Ishizu, T. Fukutomi, and T. Kakurai, Polym. J., 7, 570 (1975).

10. M. M. Miller, R. W. Amidon, and P. O. Tawney, $J$. Am. Chem. Soc., 77, 2845 (1955).

11. The Chemical Society of Japan, Ed., "Kagaku Binran, Kiso-hen II," Maruzen, Tokyo, 1975, p 575.

12. W. R. Krigbaum, J. E. Kurz, and P. Smith, J. Phys. Chem., 65, 1984 (1961).

13. J. T. Edward, J. Chem. Educ., 47, 261 (1970).

14. T. Altares, Jr., D. P. Wyman, and V. R. Allen, $J$. Polym. Sci., A, 2, 4533 (1964).

15. P. Outer, C. I. Carr, and B. H. Zimm, J. Chem. Phys., 18, 830 (1950).

16. W. R. Krigbaum and D. K. Carpenter, J. Phys. Chem., 59, 1166 (1955).

17. J. H. Wang, J. Am. Chem. Soc., 76, 4755 (1954).

18. M. Nakagawa, S. Ishida, and K. Kaneko, Kobunshi Kagaku, 29, 661 (1972).

19. K. Horie and I. Mita, Polym. J., 8, 227 (1976). 